\title{
Astrophysical water masers: Line profiles analysis
}

\author{
W. H. T. Vlemmings ${ }^{1}$ and H. J. van Langevelde ${ }^{2}$ \\ 1 Department of Astronomy, Cornell University, Ithaca, NY 14853-6801, USA* \\ e-mail: wouter@jb.man.ac.uk \\ 2 Joint Institute for VLBI in Europe, Postbus 2, 7990 AA Dwingeloo, The Netherlands
}

Received 24 November 2004 / Accepted 12 January 2005

\begin{abstract}
The changes in the spectral line profile of the $22 \mathrm{GHz} \mathrm{H}_{2} \mathrm{O}$ maser are calculated as a function of emerging maser flux. We address not only the narrowing and re-broadening of the maser lines, but also the deviations of Gaussian symmetry as a result of the various hyperfine components of the $22 \mathrm{GHz}$ maser line. Non-LTE models of the $\mathrm{H}_{2} \mathrm{O}$ maser transition, including hyperfine structure and cross-relaxation, are compared with high spectral resolution Very Long Baseline Interferometry (VLBI) observations of the $\mathrm{H}_{2} \mathrm{O}$ masers in the circumstellar envelopes (CSEs) of a sample of late-type stars. This yields estimates on the thermal width in the maser region as well as the emerging maser flux and thus the level of saturation. We also discuss the effect of a velocity gradient along the maser path on the line widths and shapes of the line profile and the effect of the geometry of the maser region. We find that the typical velocity shift along the maser path is of the order of $1.0 \mathrm{~km} \mathrm{~s}^{-1}$. The effect of this shift on the shape of the maser spectrum is difficult to distinguish from the effect of the hyperfine components.
\end{abstract}

Key words. masers - stars: circumstellar matter - stars: AGB and post-AGB - line: profiles

\section{Introduction}

The spectral line profiles of astrophysical masers contain important information on the conditions of the masing region, such as the thermal line width of the particle distribution and the optical depth. However, the degree of saturation of the maser has a strong influence on the maser profile and cannot be determined directly. It has been shown before, that a detailed analysis of the maser line widths can provide some information on the maser saturation (e.g. Goldreich \& Kwan 1974; Litvak 1970). The line profiles first get narrower with increasing radiative flux and then re-broaden when the maser becomes radiatively saturated. However, if there is an effective redistribution of the populations of the excited states due to cross-relaxation, the re-broadening will only occur until the radiative flux is large enough that the rate of stimulated emission exceeds the rate for cross-relaxation. Additionally, in the case of the $22 \mathrm{GHz}$ rotational $\mathrm{H}_{2} \mathrm{O}$ maser transition $\left(6_{16}-5_{23}\right)$, the interaction between the different hyperfine components ( $F=7-6,6-5,5-4,5-6,6-6$ and 5-5) complicates a straightforward line analysis. Several papers have addressed this issue (e.g. Nedoluha \& Watson 1991). The manifestation of the hyperfine components in the observed maser spectra also depends on the maser thermal line width, as well as the saturation and beaming angle. This is due to the fact that the hyperfine components cause significant deviations from Gaussian symmetry. The deviations can be classified by a skewness parameter.

* Based on research carried out while W.V. was based in Leiden.
In this paper we examine the feasibility of using the skewness of the profile together with the line widths to disentangle the thermal line width of the $22 \mathrm{GHz}$ masing medium and the level of saturation and beaming angle. We examine a sample of late-type stars and compare their high resolution total intensity spectra to our non-LTE models.

The $\mathrm{H}_{2} \mathrm{O}$ masers around late-type stars are thought to occur in the expanding wind at a few hundred AU from the central star (e.g. Lane et al. 1987). The temperature of the masing gas is expected to be less than $1000 \mathrm{~K}$, according to the analysis of $\mathrm{H}_{2} \mathrm{O}$ maser pumping schemes by Neufeld \& Melnick (1990). As a result the intrinsic, thermal line width of the maser should be $\lesssim 1.5 \mathrm{~km} \mathrm{~s}^{-1}$. Larger line widths can be caused by turbulence of the masing gas. Model estimates of the intrinsic thermal line widths can thus give an upper limit to the temperature in the maser region and to the effect of turbulence. At a few hundred AU from the central star the outflowing material is still being accelerated. Therefore, as shown in Rosen et al. (1978), the velocity coherent paths in the tangential and in the radial direction are of similar length. As a result, for the $\mathrm{H}_{2} \mathrm{O}$ masers neither radially nor tangentially beaming necessarily dominates. The $\mathrm{H}_{2} \mathrm{O}$ maser spots have typical sizes of $10^{13} \mathrm{~cm}$ (Reid \& Moran 1981), but as determined from VLBI observations they can be as small as a few times $10^{12} \mathrm{~cm}$ (Imai et al. 1997). Due to beaming, the actual size of the $\mathrm{H}_{2} \mathrm{O}$ maser cloud is generally much larger than the observed size. Goldreich \& Keeley (1972) have shown that for $\mathrm{H}_{2} \mathrm{O}$ masers a factor of 50 difference is likely. The maser path length could thus be as long as $10^{14}-10^{15} \mathrm{~cm}$. Along this path the velocity can change by a 
significant fraction of the intrinsic thermal line width, especially near the edge of the envelope where the velocity gradient is steep. Here we also examine the effect of such velocity changes on the shape of the $22 \mathrm{GHz} \mathrm{H_{2 }} \mathrm{O}$ maser profiles.

\section{Background}

The rate equations for the populations of the upper and lower energy levels of the various hyperfine lines of the $22 \mathrm{GHz}$ $\mathrm{H}_{2} \mathrm{O}$ maser have been solved along a uni-directional maser beam using the method described in Nedoluha \& Watson (1992, hereafter NWa) and used in Vlemmings et al. (2002, hereafter V02). We have included only the three strongest hyperfine components ( $F=7-6,6-5$ and $5-4)$, since it has previously been shown that the weakest of the hyperfine lines are negligible (Nedoluha \& Watson 1991; Deguchi \& Watson 1986). In V02, the rate equations are solved including a magnetic field, thus including all the different magnetic substates. The magnetic field does not noticeably influence the line shapes and line widths of the total intensity maser lines. Here we limit ourselves to the case of no magnetic field.

For the population levels of the upper states we solve

$$
\begin{aligned}
0= & \lambda_{i}(v)-\left(\Gamma+\Gamma_{v}\right) n_{i}(v)+R_{i j}(v)\left(n_{j}(v)-n_{i}(v)\right) \\
& +\phi(v)\left(\frac{\Gamma_{v}}{\sum g_{i}}\right) \int \mathrm{d} v \sum g_{i} n_{i}(v) .
\end{aligned}
$$

A similar but reversed equation is solved for the population of the lower levels.

Here $n_{i}(v)$ and $n_{j}(v)$ are the population numbers for respectively the upper $\left(6_{16}\right)$ and the lower $\left(5_{23}\right)$ rotational levels. $i$ denotes the upper level hyperfine states ( $F=7,6$ or 5$), j$ is the related lower hyperfine state $\left(F^{\prime}=6,5\right.$ or 4$)$. The population levels are solved as a function of molecular velocity $v$ and at different positions along the maser path. The statistical weights are designated by $g_{i}$ and $g_{j}$, and are from Kukolich (1969). The pump rate $\lambda_{i}(v)$ is assumed to be the same for the different hyperfine components and has a Maxwellian distribution. The results depend on the the ratio $\Delta \lambda / \lambda_{i}$ where $\Delta \lambda$ is the difference between the pump rate into the upper and lower levels. This ratio is of the order of a few percent (Anderson \& Watson 1993). The rate for stimulated emission $R_{i j}$ is calculated using the local maser intensity and the hyperfine interaction coefficients as described in NWa. $\Gamma$ is the decay rate for the molecular excitations. The cross-relaxation rate $\Gamma_{v}$ describes the reabsorbtion of previously emitted infrared pump photons trapped in optically thick transitions of the maser system, which generate a newly excited molecule at random, within a Maxwellian velocity distribution $(\phi(v))$. This has the effect of postponing the rebroadening of the maser line due to maser saturation. The rate for redistribution in velocity is not exactly the same for the redistribution among the different hyperfine components. However, both are expected to be roughly equal to the inverse lifetime of the state due to emission of infrared radiation. In $\mathrm{NWa}$ it was shown that the results scale by $\left[\mathrm{flux} /\left(\Gamma+\Gamma_{v}\right)\right]$. Most of the calculations are performed for $\left[\Gamma+\Gamma_{v}\right]=1.0 \mathrm{~s}^{-1}$ although we also investigate higher values of $\Gamma$ and $\Gamma_{v}$. A similar effect as the cross-relaxation due to reabsorbtion is produced

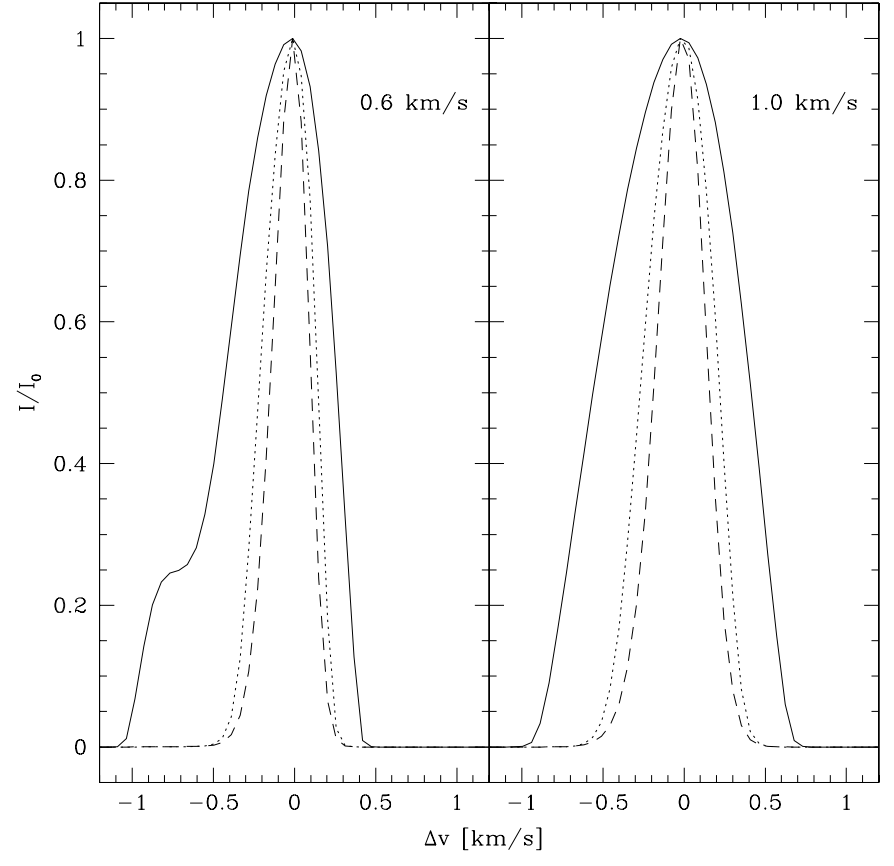

Fig. 1. Scaled intensity line profiles for the non-LTE models with $v_{\text {th }}=$ $0.6 \mathrm{~km} \mathrm{~s}^{-1}$ (left) and $v_{\mathrm{th}}=1.0 \mathrm{~km} \mathrm{~s}^{-1}$ (right). From inside out, the dashed line has emerging maser flux $T_{\mathrm{b}} \Delta \Omega=10^{8} \mathrm{~K}$ sr, the shortdashed line has $T_{\mathrm{b}} \Delta \Omega=10^{10} \mathrm{~K}$ sr and the solid line has $T_{\mathrm{b}} \Delta \Omega=$ $10^{11} \mathrm{~K}$ sr.

by elastic collisions between the maser molecules and intermixed $\mathrm{H}_{2}$ molecules, that change the velocity but not the energy of the colliding particles. This effect is described in detail in Elitzur (1990), where it was shown that due to elastic collisions, the narrowing of the maser line can be extended significantly. However, the analysis by Elitzur (1990) indicates that for maser transitions that have large infrared optical depths, such as the $\mathrm{H}_{2} \mathrm{O}$ maser discussed here, the effect of elastic collisions is small compared to that of $\Gamma$ and $\Gamma_{v}$.

Equation (1) is iteratively solved along the maser beam, as the maser intensity itself, determined using the radiative transfer equations in NWa, depends on the level populations. We assume nearly one-dimensional maser propagation, with the beaming of the maser radiation represented by the solid angle $\Delta \Omega$. The emerging maser fluxes will thus be represented in $T_{\mathrm{b}} \Delta \Omega$, with $T_{\mathrm{b}}$ the brightness temperature. The beaming angle $\Delta \Omega$ is not a constant property throughout the entire maser regime. It depends on the maser optical depth $|\tau|$ as beaming is more pronounced for the stronger masers. The beaming angle in the unsaturated cases is roughly proportional to $|\tau|^{-1}$, while in the saturated case it is proportional to $|\tau|^{-2}$ (Elitzur 1992). All calculations were performed with $T_{\mathrm{b}} \Delta \Omega=0.1 \mathrm{~K} \mathrm{sr}$, for the radiation incident onto the maser region. It was verified in $\mathrm{NWa}$ and V02 that the results are insensitive to the chosen value.

\subsection{Profile shapes}

In Fig. 1, we show an example of the line profiles resulting from our calculations. The example profiles have been calculated for two different values of the FWHM thermal width $\left(v_{\text {th }}\right)$ of the Maxwellian particle distribution. Assuming a kinetic 


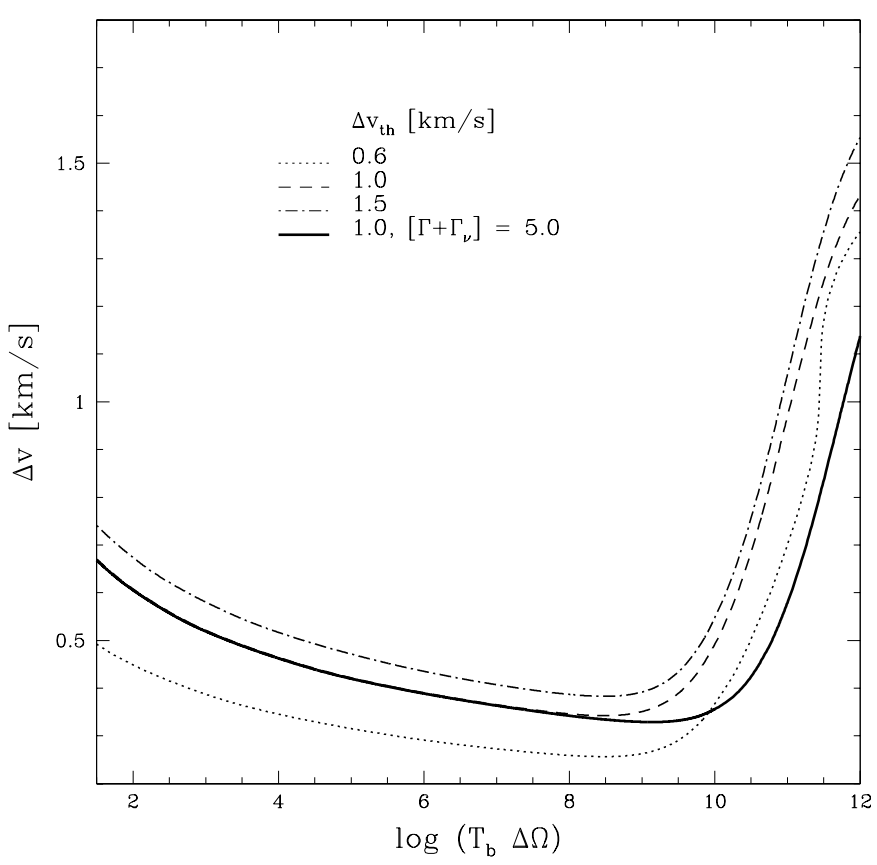

Fig. 2. Line width as a function of emerging maser flux $T_{\mathrm{b}} \Delta \Omega$ for models with different thermal width. The thick solid line is the model for $v_{\text {th }}=1.0 \mathrm{~km} \mathrm{~s}^{-1}$ with $\left[\Gamma+\Gamma_{v}\right]=5.0$, the other lines are for $\left[\Gamma+\Gamma_{v}\right]=1.0$.

temperature $T$ in Kelvin, $v_{\text {th }} \approx 0.5 \times(T / 100)^{1 / 2}(\mathrm{NWa})$. Line profiles are presented for different values of emerging flux $\left(T_{\mathrm{b}} \Delta \Omega=10^{8}, 10^{10}\right.$ and $\left.10^{11}\right)$. Due to the hyperfine interaction the maser profiles show clear deviations from Gaussian symmetry with increasing maser brightness. The effects are largest for the smallest thermal widths. We also notice the rebroadening of the profiles when the maser starts to get saturated. This can also be seen in Fig. 2, which shows the maser line width as a function of emerging flux. In the unsaturated case the maser gets increasingly narrow, when the masers starts to get saturated the line width increases. The figure shows the relations for $\left[\Gamma+\Gamma_{v}\right]=1$ and $5 \mathrm{~s}^{-1}$. As expected, the maser is able to remain unsaturated longer, for higher values of the decay and cross-relaxation rate.

Aside from the narrowing and re-broadening of the maser lines, Fig. 1 shows that due to the contributions of the hyperfine components, the shape of the $22 \mathrm{GHz} \mathrm{H}_{2} \mathrm{O}$ maser profile is also a good indicator of the saturation level. The hyperfine components will cause the profile to be skewed toward the negative velocities and the skewness will depend on the level of saturation. The deviation from Gaussian shape can be quantified by the skewness parameter defined as:

Skewness $=\frac{1}{\Psi} \int \mathrm{d} v\left[\psi(v) \frac{\left(v-v_{0}\right)}{\sigma}\right]^{3}$.

Here $\psi(v)$ is the profile of the maser line, $\sigma$ the standard deviation and $v_{0}$ the position of maximum intensity. The parameter is scaled by $\Psi$, which is the area under the profile. A positive value of the skewness parameter signifies a distribution with an asymmetric tail out toward positive velocity, while a negative skewness parameter indicates a negative tail. Figure 3 shows

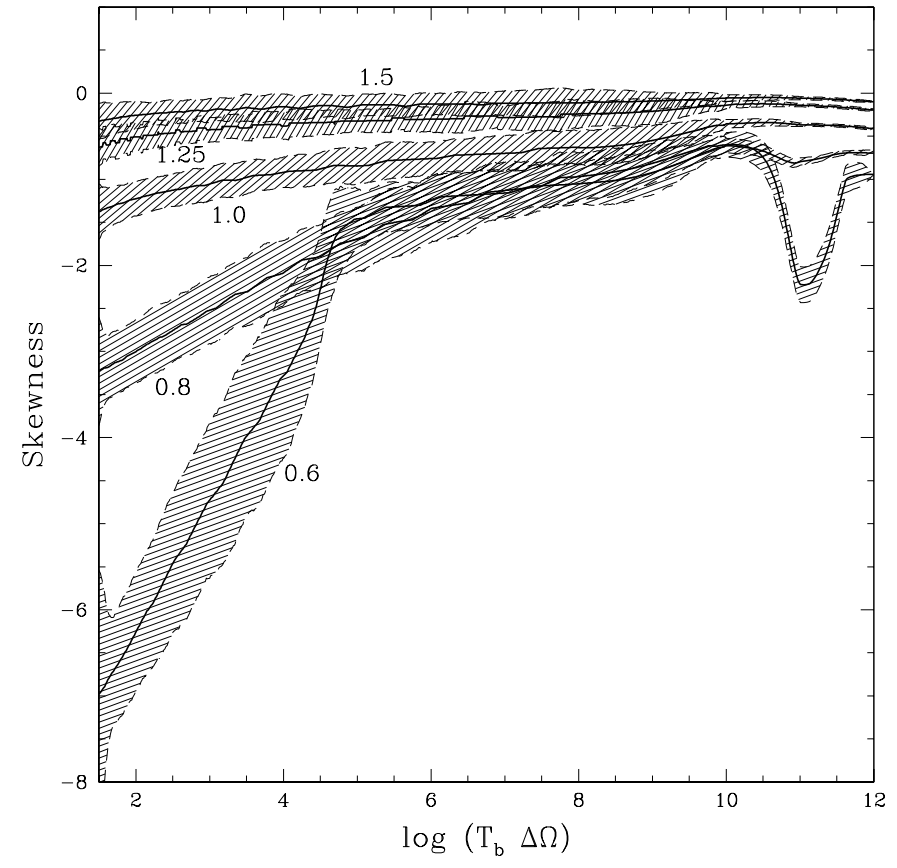

Fig. 3. Skewness parameter as a function of emerging maser brightness $T_{\mathrm{b}} \Delta \Omega$. The lines are labeled for different thermal width. The shaded areas indicate the $3 \sigma$ errors when including observational errors as described in the text.

the skewness of the maser profile as a function of emerging brightness for different values of $v_{\text {th }}$, as determined from our models. For the highly unsaturated masers the skewness parameter is strongly negative due to the multiple hyperfine components. The profiles become more symmetric when the $F=7-6$ hyperfine component starts to dominate. When the maser starts to saturate, at $T_{\mathrm{b}} \Delta \Omega \approx 10^{10}$ for $\left[\Gamma+\Gamma_{v}\right]=1 \mathrm{~s}^{-1}$, the skewness parameter decreases again, because the $F=7-6$ hyperfine component is the first to saturate. As a result, the profile becomes more asymmetric. This is the most pronounced for the lowest values of $v_{\mathrm{th}}$. For $v_{\mathrm{th}}<1.0 \mathrm{~km} \mathrm{~s}^{-1}$ the skewness parameter increases again for $T_{\mathrm{b}} \Delta \Omega>10^{11}$ after a strong decrease. For the higher thermal velocity widths the skewness parameter keeps decreasing slightly in the saturated regime.

Because the skewness parameter is the third moment of the maser profile, it is strongly affected by observational errors. To include the influence of the errors, we have simulated our observation by adding Gaussian distributed errors on top of the model profiles. We have used a signal-to-noise of 100, typical for the weakest of the observed maser sources. The result is indicated with the shaded areas in Fig. 3, these correspond to $3 \sigma$ errors. The effect of the errors is strongest in the unsaturated regime and decreases when the maser saturates. The spectral resolution of the maser profiles also affects the errors in the skewness determination. Higher spectral resolution observations have smaller errors because of a more accurate determination of the scaling parameter $\Psi$ and velocity $v_{0}$ of the peak intensity. Our models were determined with a spectral resolution of $0.027 \mathrm{~km} \mathrm{~s}^{-1}$, similar to the observations. 


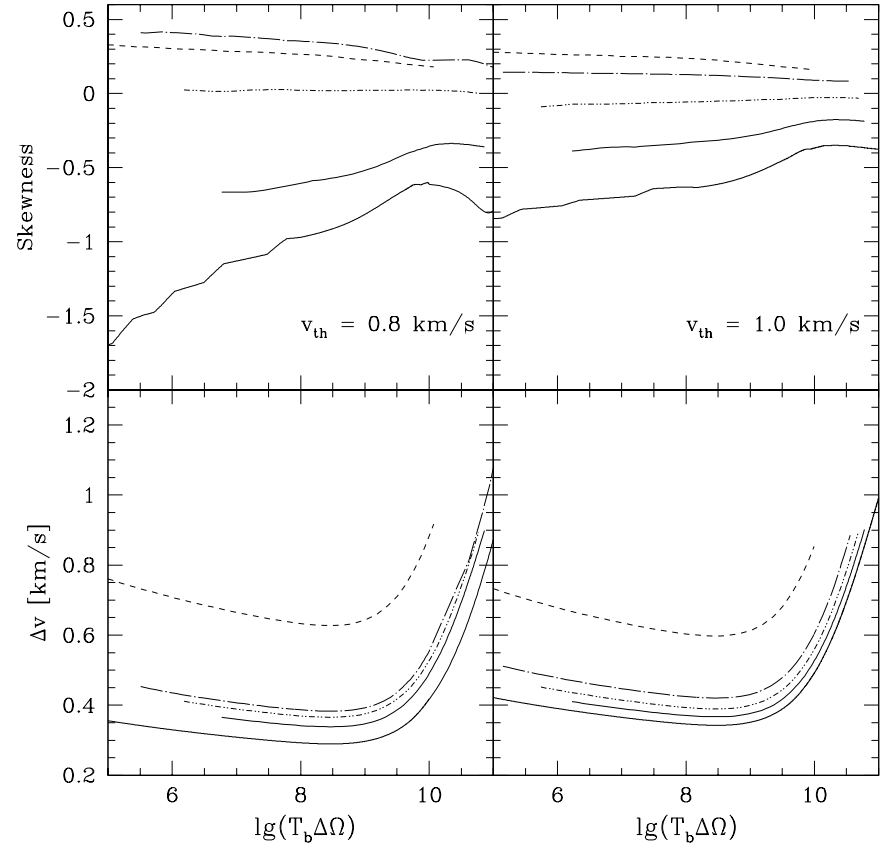

Fig. 4. Skewness and line widths for from $v_{\text {th }}=0.8$ (left) and $1.0 \mathrm{~km} \mathrm{~s}^{-1}$ (right) when including a velocity shift $\Delta V_{\mathrm{m}}$ of 0.4 (thin solid), 0.6 (short-dashed), 0.8 (dashed-dotted) and $1.2 \mathrm{~km} \mathrm{~s}^{-1}$ (longdashed) as a function of emerging maser brightness $T_{\mathrm{b}} \Delta \Omega$. The thick solid line indicates the result with constant velocity along the maser path. Due to the computational requirements we have not calculated the full brightness temperature range for the models which have a velocity gradient.

\subsection{Velocity gradients}

Obviously the line profile can also be determined by velocity gradients along the radiation path, giving rise to observable skewness. Therefore, the calculations above have also been performed including a shift of velocity along the maser path $\left(\Delta V_{\mathrm{m}}\right)$. This has been done by replacing the pump rate $\lambda(v)$ in Eq. (1) for all hyperfine components by $\lambda(v+\delta v)$, where $\delta v$ increases for every step along the maser path. The effects have been calculated for the models with $v_{\text {th }}=0.8$ and $1.0 \mathrm{~km} \mathrm{~s}^{-1}$. Results for the line widths and skewness parameter are shown in Fig. 4 for $\Delta V_{\mathrm{m}}$ of up to $1.2 \mathrm{~km} \mathrm{~s}^{-1}$. In our calculations, the linear velocity gradient along the maser path is characterized by $\Delta V_{\mathrm{m}}$, the velocity shift between the start and the end of the path. The small variations on the lines are due to the spectral resolution of our models, which is equal to the resolution of the observations.

The skewness is very sensitive to $\Delta V_{\mathrm{m}}$, especially in the unsaturated case. The effect of $\Delta V_{\mathrm{m}}$ becomes less for models with a relatively large thermal width. When $\Delta V_{\mathrm{m}}$ becomes significantly larger than the thermal width, the skewness no longer depends on the intrinsic width. This explains that, in Fig. 4, the model for $v_{\text {th }}=0.8$ and a $\Delta V_{\mathrm{m}}=1.2 \mathrm{~km} \mathrm{~s}^{-1}$ is less positively skewed than the model with $\Delta V_{\mathrm{m}}=0.8 \mathrm{~km} \mathrm{~s}^{-1}$, while this is not seen in the models with $v_{\text {th }}=1.0$. Most models show less deviations of Gaussian symmetry in the saturated case. All the models in Fig. 4 were calculated with a negative velocity gradient along the maser path. A gradient in the positive velocity direction increases the negative skewness of the profiles for all cases. Since the front side of the envelope has the most negative velocity, the velocity gradient through the envelope toward the observer is predominantly negative, for features originating from both sides of the circumstellar envelope.

We find that a velocity gradient along the maser path increases the maser line width with an approximately equal amount in both the unsaturated as well as the saturated case. As soon as $\Delta V_{\mathrm{m}}$ approaches the intrinsic thermal line width, the measured line widths increase rapidly. Nedoluha \& Watson (1988) have shown that for $\Delta V_{\mathrm{m}}$ a few times $v_{\mathrm{th}}$, the maser line splits into several distinct, narrow features, each becoming an independent maser. This has been observed for $\mathrm{H}_{2} \mathrm{O}$ masers in star-forming regions (e.g. Genzel et al. 1979; Walker 1984). Our observations of the circumstellar $\mathrm{H}_{2} \mathrm{O}$ masers do not show this line splitting, indicating that the largest velocity shift along the maser path is not more than $\approx 2.0 \mathrm{~km} \mathrm{~s}^{-1}$.

\section{Observations}

The observations were performed at the $\mathrm{NRAO}^{1}$ Very Long Baseline Array (VLBA) at December 13th 1998 as part of the project designed to measure the circular polarization of circumstellar $\mathrm{H}_{2} \mathrm{O}$ masers. The results of the polarization measurements are presented in V02. At the frequency of the $6_{16}-5_{23}$ rotational transition of $\mathrm{H}_{2} \mathrm{O}, 22.235 \mathrm{GHz}$, the average beam width is $\approx 0.5 \times 0.5$ mas. This allows us to resolve the different $\mathrm{H}_{2} \mathrm{O}$ maser features in the CSE. As explained in V02, the data were correlated twice, once with modest $(7.8 \mathrm{kHz}=$ $0.1 \mathrm{~km} \mathrm{~s}^{-1}$ ) spectral resolution, and once with high spectral resolution $\left(1.95 \mathrm{kHz}=0.027 \mathrm{~km} \mathrm{~s}^{-1}\right)$. In this paper we use the total intensity profiles obtained with the high spectral resolution data. We have performed $6 \mathrm{~h}$ of observations per sourcecalibrator pair. The calibrator was observed for $11 / 2 \mathrm{~h}$ in a number of scans equally distributed over the $6 \mathrm{~h}$. We used 2 filters of $1 \mathrm{MHz}$ width, which were overlapped to get a velocity coverage of $\approx 22 \mathrm{~km} \mathrm{~s}^{-1}$. This covers most of the velocity range of the $\mathrm{H}_{2} \mathrm{O}$ maser.

\subsection{Sample}

We observed 4 late type stars, the supergiants S Per, VY CMa and NML Cyg and the Mira variable star U Her. They are listed in Table 1 with type, position, distance and period. The sources were selected on the basis of 2 criteria; strong $\mathrm{H}_{2} \mathrm{O}$ masers and the availability of $\mathrm{SiO}$ and/or $\mathrm{OH}$ maser polarization observations. The peak fluxes measured in the observations are shown in Table 1 . On the total intensity maps the noise is between $\approx 0.08-0.3 \mathrm{Jy}$.

\section{Results}

The results of the observations are shown in Table 2. The table gives the LSR velocity of the maser feature, the maser strength

1 The National Radio Astronomy Observatory is a facility of the National Science Foundation operated under cooperative agreement by Associated Universities, Inc. 
Table 1. Star sample.

\begin{tabular}{lccccccc}
\hline \hline Name & Type & $\begin{array}{c}\text { RA } \\
(\mathrm{h} \mathrm{m} \mathrm{s})\end{array}$ & $\begin{array}{c}\text { Dec } \\
\left({ }^{\prime \prime \prime \prime}\right)\end{array}$ & $\begin{array}{c}\text { Distance } \\
(\mathrm{pc})\end{array}$ & $\begin{array}{c}\text { Period } \\
(\text { days })\end{array}$ & $\begin{array}{c}V_{\text {rad }} \\
\left(\mathrm{km} \mathrm{s}^{-1}\right)\end{array}$ & $\begin{array}{c}\text { Peak flux } \\
(\mathrm{Jy})\end{array}$ \\
\hline S Per & Supergiant & 022251.72 & +583511.4 & 1610 & 822 & -38.1 & 76.1 \\
U Her & Mira & 162547.4713 & +185332.867 & 277 & 406 & -14.5 & 12.5 \\
NML Cyg & Supergiant & 204625.7 & +400656 & 1220 & 940 & 0.0 & 48.2 \\
VY CMa & Supergiant & 072258.3315 & -254603.174 & 1500 & 2000 & 22.0 & 244.1 \\
\hline
\end{tabular}

Table 2. Results.

\begin{tabular}{|c|c|c|c|c|c|c|}
\hline Name & $\begin{array}{c}V_{\text {rad }} \\
\left(\mathrm{km} \mathrm{s}^{-1}\right)\end{array}$ & $\begin{array}{c}\text { Flux }(I) \\
(\mathrm{Jy})\end{array}$ & $\begin{array}{c}\Delta v_{\mathrm{L}} \\
\left(\mathrm{km} \mathrm{s}^{-1}\right)\end{array}$ & Skewness & $3 \sigma_{\text {skew }}^{+}$ & $3 \sigma_{\text {skew }}^{-}$ \\
\hline \multirow[t]{12}{*}{ S Per } & -23.2 & 10.0 & 0.44 & -0.29 & +0.11 & -0.24 \\
\hline & -27.2 & 76.1 & 0.88 & -2.21 & +0.31 & -0.43 \\
\hline & -27.2 & 14.2 & 0.51 & 0.04 & +0.06 & -0.10 \\
\hline & -28.9 & 29.7 & 0.61 & 4.35 & +0.84 & -0.66 \\
\hline & -30.4 & 15.4 & 0.92 & -0.10 & +0.04 & -0.09 \\
\hline & -30.9 & 57.7 & 0.77 & $-0.73^{*}$ & +0.11 & -0.16 \\
\hline & -33.9 & 11.4 & 1.21 & -0.59 & +0.10 & -0.15 \\
\hline & -34.2 & 14.9 & 0.48 & -0.20 & +0.06 & -0.14 \\
\hline & -37.1 & 34.7 & 0.54 & -0.25 & +0.05 & -0.09 \\
\hline & -39.2 & 26.6 & 0.64 & 1.31 & +0.27 & -0.22 \\
\hline & -39.3 & 12.9 & 0.69 & -0.17 & +0.06 & -0.11 \\
\hline & -40.1 & 35.6 & 0.53 & -0.19 & +0.04 & -0.06 \\
\hline \multirow[t]{10}{*}{ VY CMa } & 10.6 & 155.5 & 0.67 & $-0.35^{*}$ & +0.05 & -0.09 \\
\hline & 10.8 & 244.1 & 0.66 & $-0.53^{*}$ & +0.08 & -0.11 \\
\hline & 12.9 & 115.2 & 0.74 & -1.19 & +0.17 & -0.25 \\
\hline & 13.7 & 47.9 & 0.83 & -0.11 & +0.02 & -0.04 \\
\hline & 15.0 & 17.8 & 0.94 & 0.01 & +0.02 & -0.05 \\
\hline & 18.3 & 58.5 & 0.80 & -0.36 & +0.06 & -0.08 \\
\hline & 24.6 & 126.2 & 0.60 & 0.07 & +0.02 & -0.03 \\
\hline & 25.2 & 65.6 & 0.79 & 0.34 & +0.07 & -0.06 \\
\hline & 27.1 & 25.3 & 0.88 & 0.22 & +0.06 & -0.06 \\
\hline & 28.8 & 43.1 & 0.61 & -0.32 & +0.05 & -0.06 \\
\hline \multirow[t]{4}{*}{ NML Cyg } & -20.0 & 3.66 & 0.54 & 0.64 & +0.32 & -0.36 \\
\hline & -20.2 & 5.42 & 0.54 & -0.14 & +0.11 & -0.30 \\
\hline & -20.4 & 10.5 & 0.40 & -0.09 & +0.08 & -0.19 \\
\hline & -21.2 & 48.2 & 0.66 & -0.03 & +0.02 & -0.03 \\
\hline \multirow[t]{6}{*}{ U Her } & -17.6 & 2.91 & 0.50 & 5.47 & +1.65 & -1.60 \\
\hline & -17.6 & 1.16 & 0.73 & 0.69 & +0.55 & -0.63 \\
\hline & -17.8 & 12.20 & 0.75 & 0.69 & +0.18 & -0.16 \\
\hline & -19.2 & 2.46 & 0.47 & -0.63 & +0.38 & -0.93 \\
\hline & -19.2 & 2.07 & 0.51 & -0.14 & +0.33 & -0.76 \\
\hline & -19.3 & 1.36 & 0.51 & -0.27 & +0.46 & -1.14 \\
\hline
\end{tabular}

${ }^{*}$ Bad spectral channels affect skewness determination. 

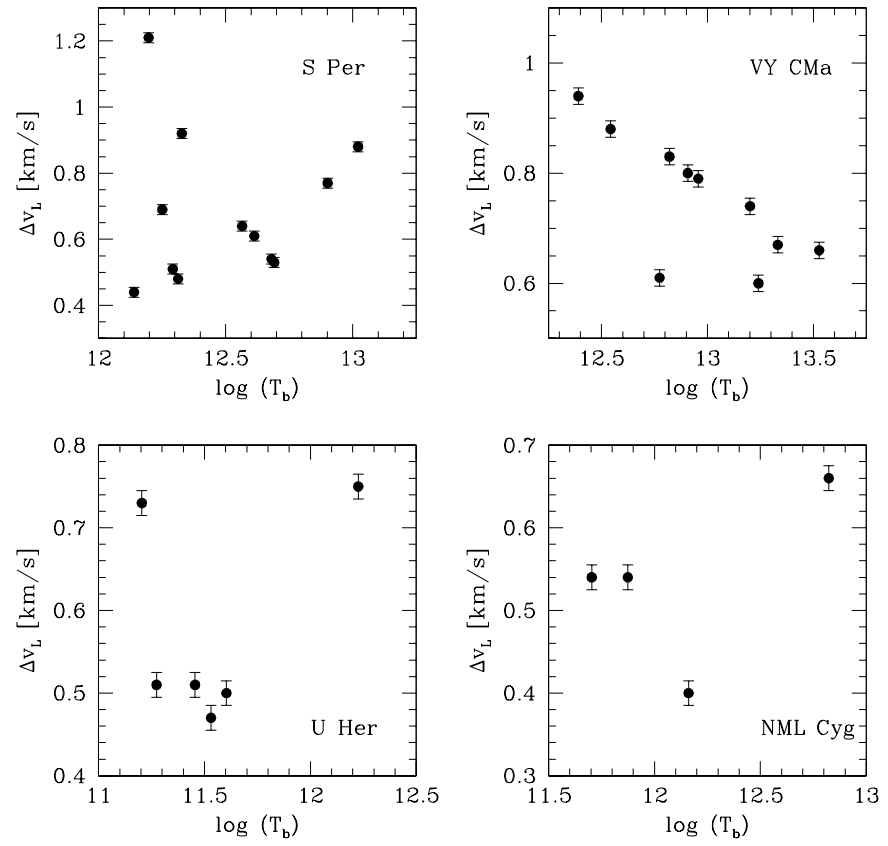

Fig. 5. Line widths of the observed maser features as a function of emerging flux. The error bars are the $3 \sigma$ errors.

in Jy and the line width and skewness parameter. The features labeled * have a few bad spectral channels in the wings of the profile due to interference. This can have a significant effect on the skewness of the profile; we have verified that the values listed are the upper limits for these features and the actual skewness could be up to $\approx 1.0$ lower. The quoted $3 \sigma$ error values of the skewness, are based on the errors in the determination of $v_{0}$ and $\sigma$ for use in Eq. (2). These depend on the signal-tonoise ratio of the maser feature, and on the spectral resolution of the observations.

We will plot the observed line width and skewness as a function of brightness temperature in order to make a comparison with our models. One should realize that there is not necessarily a single value for width and velocity shift that is valid for all maser features in any star. It is natural to expect the results to be quite scattered.

Figures 5 and 6 show the observed line widths $\Delta v_{L}$ and Skewness parameters plotted against the logarithm of the maser brightness temperature $T_{\mathrm{b}}$. This is calculated using the fluxes listed in Table 2 using the beam size of the observations as the maser spots are unresolved, thus $T_{\mathrm{b}}$ is formally only a lower limit. However, at the distance of the star the beam has a diameter of approximately $1-2 \times 10^{13} \mathrm{~cm}$ and is comparable to the typical maser spot size (Reid \& Moran 1981). We thus expect the determined brightness temperatures to be 0 with the real values.

\section{1. $U$ Her}

For the Mira variable star U Her most of the maser features show line widths of $\approx 0.5 \mathrm{~km} \mathrm{~s}^{-1}$ with 2 of the features showing widths of $\approx 0.75 \mathrm{~km} \mathrm{~s}^{-1}$. There is no clear trend with increasing maser brightness. The $\mathrm{H}_{2} \mathrm{O}$ masers around $\mathrm{U}$ Her have also
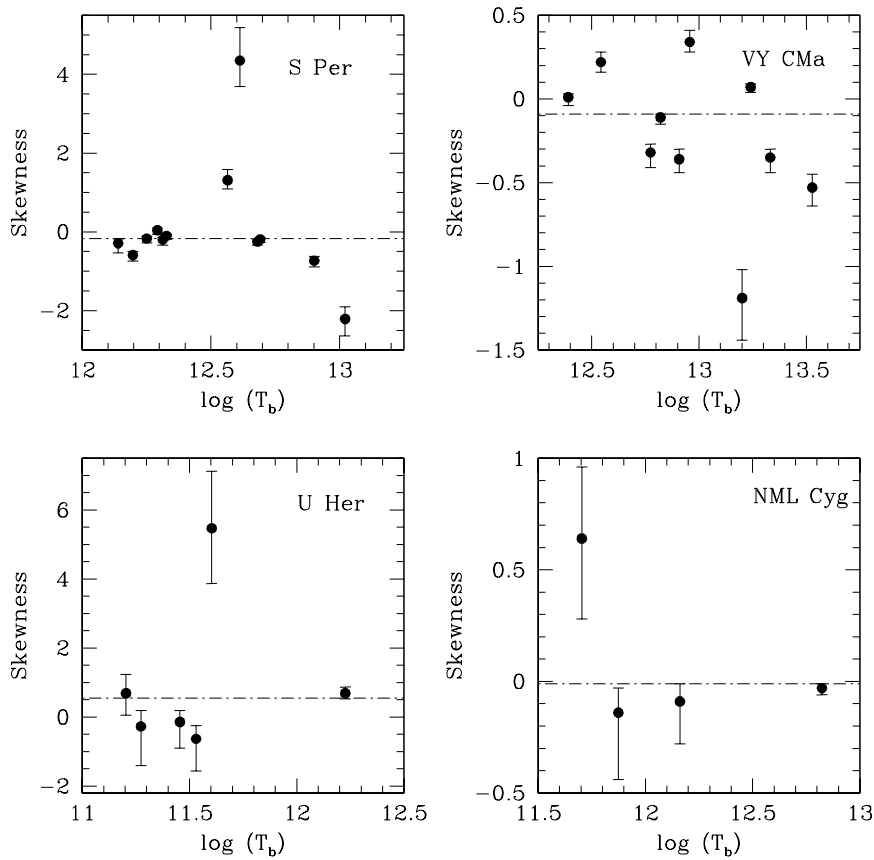

Fig. 6. Skewness parameter of the observed maser features as a function of emerging flux. The error bars indicate $3 \sigma$ errors and the dashed-dotted line is the weighted average.

been observed with the Very Large Array (VLA) by Colomer et al. (2000). They observe line widths several times wider than found in our observations, but the VLA is unable to resolve the individual spots and they only used a spectral resolution of $0.33 \mathrm{~km} \mathrm{~s}^{-1}$. It is likely that the larger line widths are due to the blending of several smaller components.

Because U Her has the weakest masers, the errors on the skewness parameter are relatively large. The weighted average of the skewness is 0.55 , a possible indication of large $\Delta V_{\mathrm{m}}$. The skewness is positive for the maser features with $V_{\mathrm{LSR}} \approx$ $-17.5 \mathrm{~km} \mathrm{~s}^{-1}$, while it is negative for the features with $V_{\mathrm{LSR}} \approx$ $-19.0 \mathrm{~km} \mathrm{~s}^{-1}$. All the maser features are blue-shifted with respect to the stellar velocity of $V_{\mathrm{LSR}}=-14.4 \mathrm{~km} \mathrm{~s}^{-1}$. As discussed above, $\Delta V_{\mathrm{m}}$ will be negative because the maser exists in a radially accelerating outflow. This results in a frequency shift along the maser feature in the direction of the hyperfine components. If the features with a velocity closer to the stellar velocity are located closer to the star, they will experience more acceleration along the maser path and thus a larger velocity gradient. This can explain that the skewness of these features is more positive than the that of the other features. The maser feature with the skewness of 5.47 however, is likely a blend of two overlapping maser features.

\subsection{NML Cyg}

The line widths of the maser features around NML Cyg are found to lie between 0.4 and $0.7 \mathrm{~km} \mathrm{~s}^{-1}$. Again no clear trend can be determined, especially since we have only been able to detect 4 maser spots. MERLIN observations by Richards et al. (1996) show a much more complex structure with many 
features. The line widths they find cover a large range between 0.4 and $2.5 \mathrm{~km} \mathrm{~s}^{-1}$.

The weighted average of the skewness is -0.01 . The line widths and skewness, indicate an intrinsic thermal width of between 0.8 and $1.0 \mathrm{~km} \mathrm{~s}^{-1}$ and $\Delta V_{\mathrm{m}}$ of $\approx 0.75 \mathrm{~km} \mathrm{~s}^{-1}$. A larger $\Delta V_{\mathrm{m}}$ would result in a more positive skewness, while a larger thermal width does not allow for the observed narrow line widths.

\section{3. $S$ Per}

The line widths of the maser features around S Per show some indication of an increase with maser brightness. The line width increases from 0.4 to 0.9 and varies approximately as $\Delta v \propto\left(T_{\mathrm{b}}\right)^{0.3}$. However, 2 features show a width which is higher than expected. But, as seen in Fig. 4, this can be due to a velocity shift along the path which is more than $1.2 \mathrm{~km} \mathrm{~s}^{-1}$. Alternatively, the actual spot size of these features could be less than $10^{12} \mathrm{~cm}$ leading to an underestimation of $T_{\mathrm{b}}$. The skewness of the other features indicate a $\Delta V_{\mathrm{m}}$ of not more than $0.8 \mathrm{~km} \mathrm{~s}^{-1}$. Similar velocity shifts have been observed by Richards et al. (1999). The observed line widths correspond to a thermal line width between 0.8 and $1.0 \mathrm{~km} \mathrm{~s}^{-1}$.

A combination of both the skewness and line width analysis allows us to estimate, from our models, the actual emerging maser brightness temperature and beaming angle. For the estimated velocity shifts along the maser path and thermal line widths, our models give observed line widths of $\approx 0.4-0.9 \mathrm{~km} \mathrm{~s}^{-1}$ for $T_{\mathrm{b}} \Delta \Omega$ between $10^{10}$ and $10^{11} \mathrm{~K}$ sr. With observed brightness temperature $T_{\mathrm{b}}=10^{12}-10^{13} \mathrm{~K}$, this indicates that the beaming solid angle $\Delta \Omega \approx 10^{-2}$ sr.

\subsection{VY CMa}

The line widths of the $\mathrm{H}_{2} \mathrm{O}$ masers around VY CMa show a clear dependence on the brightness temperature. The line width varies with $\Delta v \propto\left(T_{\mathrm{b}}\right)^{-\alpha}$, where $\alpha \approx 0.3$. A similar decrease of line width with intensity was observed before on the maser flare in Cepheus A (Rowland \& Cohen 1986), though there $\alpha \approx$ 0.5 . We observe line widths from 0.6 to $0.94 \mathrm{~km} \mathrm{~s}^{-1}$. MERLIN observations (Richards et al. 1999) have found many features to have a velocity extent of up to $3 \mathrm{~km} \mathrm{~s}^{-1}$, indicating the presence of large velocity shifts along the maser.

For VY CMa, the skewness and line widths indicate a thermal width in the $\mathrm{H}_{2} \mathrm{O}$ maser region of $\approx 1.0-1.2 \mathrm{~km} \mathrm{~s}^{-1}$, with $\Delta V_{\mathrm{m}}$ up to $1.25 \mathrm{~km} \mathrm{~s}^{-1}$. Using this, according to our models, $T_{\mathrm{b}} \Delta \Omega$ is between $10^{10}$ to $10^{11} \mathrm{~K}$ sr, while the observed lower limit for $T_{\mathrm{b}} \approx 10^{12.4} \mathrm{~K}$. This indicates a beaming solid angle of $\approx 10^{-3} \mathrm{sr}$.

\section{Discussion}

The non-LTE method used here to model the shape of the $22 \mathrm{GHz} \mathrm{H}_{2} \mathrm{O}$ maser spectrum was previously used to determine the circular polarization of the maser in the presence of a magnetic field (V02). There the non-LTE modeling method, is compared with a standard LTE method which is described in Vlemmings et al. (2001). The non-LTE approach was developed to analyze circular polarization spectra and successfully reproduced the distribution over magnetic components. In principle, similar physical processes are important for understanding the line shapes of the $\mathrm{H}_{2} \mathrm{O}$ maser blend and we apply the same analysis here. Recently, Watson et al. (2002) have used a similar method to examine the line shapes of interstellar $\mathrm{H}_{2} \mathrm{O}$ masers, although their analysis has been based on only a two-level maser transition, thus only 1 hyperfine component is included. Instead of using the skewness of the maser profile, they examine the second order deviations of Gaussian symmetry, the "kurtosis". They find that the interstellar $\mathrm{H}_{2} \mathrm{O}$ masers are found in gas with temperatures greater than $1200 \mathrm{~K}$, for which the influence of multiple hyperfine components is thought to be small. Since the gas in CSEs is at a lower temperature (e.g. Goldreich \& Scoville 1976), the effect of the other hyperfine components is larger, as is indeed seen our results.

The large spread in the observed skewness parameter indicates that velocity gradients along the maser path play a large role in determining the shape of the $\mathrm{H}_{2} \mathrm{O}$ maser line profiles. $35 \%$ of the maser features have a positive skewness, which only occurs for masers that are starting to saturate and which have a relatively large negative $\Delta V_{\mathrm{m}}$.

The observed $\Delta v \propto\left(T_{\mathrm{b}}\right)^{-\alpha}$ dependence, with different values of $\alpha$ ( $\alpha \approx-0.3$ for S Per and 0.3 for VY CMa), can be explained by the relation between beaming angle $\Delta \Omega$ and maser optical depth $|\tau|$. As noted before, in the completely unsaturated case $\Delta \Omega \propto|\tau|^{-1}$, while this increases to $\Delta \Omega \propto|\tau|^{-2}$ in the saturated case. Additionally, in the unsaturated regime the amplification is exponential, so $T_{\mathrm{b}} \propto \mathrm{e}^{|\tau|}$, while in the saturated regime the amplification becomes linear and $T_{\mathrm{b}} \propto|\tau|$. This means that, while unsaturated, the beaming angle $\Delta \Omega \propto\left(\ln T_{\mathrm{b}}\right)^{-1}$, and when saturated $\Delta \Omega \propto T_{\mathrm{b}}^{-2}$. Meanwhile, our models in Fig. 2 show, that before the maser starts re-broadening the line width is almost constant with increasing $T_{\mathrm{b}} \Delta \Omega$. after rebroadening, when saturation occurs, the line widths becomes approximately proportional to $\left(T_{\mathrm{b}} \Delta \Omega\right)^{0.5}$. Using the results for the variation of $\Delta \Omega$ with $T_{\mathrm{b}}$ we then find that for unsaturated masers $\Delta v \propto\left(T_{\mathrm{b}}\right)^{0.7}$, while the line width of saturated masers will be proportional to $\left(T_{\mathrm{b}}\right)^{-0.5}$. The value of $\alpha$ can thus increase from $\approx-0.7$ in the mostly unsaturated regime to 0.5 in the saturated regime. This seems to indicate that the masers around S Per are still mostly unsaturated, while those around VY CMa are close to becoming saturated. However, the presence of velocity shifts along the maser path complicates this straightforward explanation. For example, if the high brightness maser features trace the lower velocity shifts because a strong gradient disrupts the maser growth, we can also observe a decrease of the line width with increasing brightness. Additionally, velocity shifts will introduce a big spread in the relation between line width and maser brightness.

An additional uncertainty in the analysis is introduced by assuming a linear maser model. While astrophysical masers, and especially $\mathrm{H}_{2} \mathrm{O}$ masers, are thought to be highly elongated, the actual three-dimensional shape of the maser will affect the maser line width and shape as described by Elitzur (1994). There it was shown that the linear maser model is sufficient 
to describe the maser profile out to $\sim 2 v_{\text {th }}$ for spherical and $\sim 3-5 v_{\text {th }}$ for elongated cylindrical masers. However, crossing maser beams will suppress the emission in the outer wings of the maser profile. This can cause narrowing of the maser line to be observed even for saturated masers. In the case of the objects studied in this paper, the effect is expected to be small or even negligible, as the suppression of the maser profile wings only becomes significant when the maser becomes saturated. Then, as shown in Elitzur (1994), the suppression effects on the profile are only noticeable when the dynamic range of the spectrum is large. For a filamentary maser, the dynamic range has to be higher than $\approx 7 \times 10^{3}$, while the largest dynamic range for our maser sources is $\approx 7 \times 10^{2}$ for the strongest maser feature of VY CMa. If the masers were purely spherical however, the measured linewidth decrease for increasing maser brightness for the VY CMa $\mathrm{H}_{2} \mathrm{O}$ masers could be partly due to this geometric effect. As a result the estimated intrinsic thermal width and the beaming solid angle might be slightly underestimated.

The presence of large velocity gradients along the maser path, and the uncertainty in the exact maser geometry, increases the difficulty of determining underlying thermal widths, level of saturation and beaming angle. However, from comparison with the models we have been able to give estimates for most of the sources presented here. In general the thermal line width of the maser medium in our sources is between 0.8 and $1.0 \mathrm{~km} \mathrm{~s}^{-1}$. This line width can be caused by the actual temperature of the maser gas, as well as by turbulent motions. The $\mathrm{H}_{2} \mathrm{O}$ maser region should not have temperatures higher than $T \approx 1000 \mathrm{~K}$ (Neufeld \& Melnick 1990), which implies that the line widths due to the thermal motions should not be higher than $\approx 1.5 \mathrm{~km} \mathrm{~s}^{-1}$. This is consistent with our observations, which indicate a temperature of $T \approx 400-600 \mathrm{~K}$. The line width due to turbulent motions should then be of the same order of magnitude or less. This is significantly less than the turbulent width determined by Colomer et al. (2000), who give a width of the order of $2-4 \mathrm{~km} \mathrm{~s}^{-1}$. But as discussed above they found larger line widths, possibly due to the fact that the VLA was not able to resolve individual maser features.

Similar to earlier observations (e.g. Spencer et al. 1979), our observations suggest that the circumstellar $\mathrm{H}_{2} \mathrm{O}$ masers are either unsaturated or only slightly saturated, with intrinsic brightness temperatures between $T_{\mathrm{b}}=10^{11}-10^{13} \mathrm{~K}$. From the models we can estimate the typical beaming angle to be between $\Delta \Omega=10^{-2}-10^{-3} \mathrm{sr}$, similar to values found before (e.g. Nedoluha \& Watson 1991).

\section{Conclusions}

A detailed analysis of the line profiles of $22 \mathrm{GHz} \mathrm{H}_{2} \mathrm{O}$ masers can be a valuable tool to estimate saturation, thermal velocity width and velocity shift along the maser path in the maser region. Because the $22 \mathrm{GHz}$ transition consists of multiple hyperfine components, the maser profile shows clear deviations from symmetry at various stages of saturation. Unfortunately, the effect of velocity shifts along the maser path, can not easily be detached from the effects of hyperfine contributions. Additionally, for VY CMa, the actual unknown maser geometry introduces another level of complexity.

However, we have been able to obtain some estimates on the thermal line widths and beaming angles on the $\mathrm{H}_{2} \mathrm{O}$ masers around a sample of 4 late-type stars. It is clear that velocity shifts along the maser path of up to $1.25 \mathrm{~km} \mathrm{~s}^{-1}$ play an important role, and will have to be taken into account for further comparisons between models and observations. The thermal line widths in the maser regions are found to be mostly around $1.0 \mathrm{~km} \mathrm{~s}^{-1}$, indicating temperatures of approximately $400 \mathrm{~K}$ in the masing region. Most masers are thought to be only slightly saturated at the most, with $T_{\mathrm{b}} \Delta \Omega \lesssim 10^{11} \mathrm{~K} \mathrm{sr}$. Beaming solid angles for the circumstellar $\mathrm{H}_{2} \mathrm{O}$ masers are found to be typically between $10^{-2}$ and $10^{-3} \mathrm{sr}$.

Acknowledgements. W.V. thanks the Niels Stensen Foundation for partly supporting his stay at Cornell University.

\section{References}

Anderson, N., \& Watson, W. D. 1993, ApJ, 407, 620

Colomer, F., Reid, M. J., Menten, K. M., \& Bujarrabal, V. 2000, A\&A, 355,979

Deguchi, S., \& Watson, W. D. 1986, ApJ, 302, 750

Elitzur, M. 1990, ApJ, 350, L17

Elitzur, M., Astronomical Masers (Kluwer Academic Publishers, Astrophysics and Space Science Library), 170, 365

Elitzur, M. 1994, ApJ, 422, 751

Genzel, R., Downes, D., Moran, J. M., et al. 1979, A\&A, 78, 239

Goldreich, P., \& Keeley, D. A. 1972, ApJ, 174, 517

Goldreich, P., \& Kwan, J. Y. 1974, ApJ, 190, 27

Goldreich, P., \& Scoville, N. 1976, ApJ, 205, 144

Imai, H., Sasao, T., Kameya, O., et al. 1997, A\&A, 317, L67

Kukolich, S. G. 1969, J. Chem. Phys., 50, 3751

Lane, A. P., Johnston, K. J., Bowers, P. F., et al. 1987, ApJ, 323, 756

Litvak, M. M. 1970, Phys. Rev. A, 2, 2107

Nedoluha, G. E., \& Watson, W. D. 1988, ApJ, 335, L19

Nedoluha, G. E., \& Watson, W. D. 1991, ApJ, 367, L63

Nedoluha, G. E., \& Watson, W. D. 1992, ApJ, 384, 185 (NWa)

Neufeld, D. A., \& Melnick, G. J. 1990, ApJ, 352, L9

Richards, A. M. S., Yates, J. A., \& Cohen, R. J. 1996, MNRAS, 282, 665

Richards, A. M. S., Yates, J. A., \& Cohen, R. J. 1999, MNRAS, 306, 954

Reid, M. J., \& Moran, J. M. 1981, ARA\&A, 19, 231

Rosen, B. R., Moran, J. M., Reid, M. J., et al. 1978, ApJ, 222, 132

Rowland, P. R., \& Cohen, R. J. 1986, MNRAS, 220, 233

Spencer, J. H., Johnston, K. J., Moran, J. M., et al. 1979, ApJ, 230, 449

Vlemmings, W., Diamond, P. J., \& van Langevelde, H. J. 2001, A\&A, 375, L1

Vlemmings, W., Diamond, P. J., \& van Langevelde, H. J. 2002, A\&A, 394, 589 (V02)

Walker, R. C. 1984, ApJ, 280, 618

Watson, W. D., Sarma, A. P., \& Singleton, M. S. 2002, ApJ, 570, L37 